\title{
Impact of chronic administration of anabolic androgenic steroids and taurine on blood pressure in rats
}

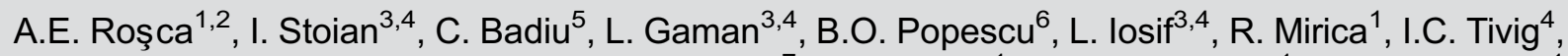 \\ C.S. Stancu ${ }^{7}$, C. Căruntu ${ }^{1}$, S.E. Voiculescu ${ }^{1}$ and L. Zăgrean ${ }^{1}$ \\ ${ }^{1}$ Department of Physiology, Carol Davila University of Medicine and Pharmacy, Bucharest, Romania \\ ${ }^{2}$ Victor Babeş National Institute of Research-Development in the Pathology Domain, Bucharest, Romania \\ ${ }^{3}$ Department of Biochemistry, Carol Davila University of Medicine and Pharmacy, Bucharest, Romania \\ ${ }^{4}$ R\&D Irist Labmed, Bucharest, Romania \\ ${ }^{5}$ Department of Endocrinology, C.I. Parhon National Institute of Endocrinology, \\ Carol Davila University of Medicine and Pharmacy, Bucharest, Romania \\ ${ }^{6}$ Department of Neurology, Colentina Clinical Hospital, Carol Davila University of Medicine and Pharmacy, Bucharest, Romania \\ ${ }^{7}$ Department of Lipoproteins and Atherosclerosis, N. Simionescu Institute of Cellular Biology and Pathology, Bucharest, Romania
}

\begin{abstract}
Supraphysiological administration of anabolic androgenic steroids has been linked to increased blood pressure. The widely distributed amino acid taurine seems to be an effective depressor agent in drug-induced hypertension. The purpose of this study was to assess the impact of chronic high dose administration of nandrolone decanoate (DECA) and taurine on blood pressure in rats and to verify the potentially involved mechanisms. The study was conducted in 4 groups of 8 adult male Wistar rats, aged 14 weeks, treated for 12 weeks with: DECA (A group); vehicle (C group); taurine (T group), or with both drugs (AT group). Systolic blood pressure (SBP) was measured at the beginning of the study $\left(\mathrm{SBP}_{1}\right), 2\left(\mathrm{SBP}_{2}\right)$ and 3 months $\left(\mathrm{SBP}_{3}\right)$ later. Plasma angiotensin-converting enzyme (ACE) activity and plasma end products of nitric oxide metabolism $\left(\mathrm{NO}_{\mathbf{x}}\right)$ were also determined. $\mathrm{SBP}_{3}$ and $\mathrm{SBP}_{2}$ were significantly increased compared to $\mathrm{SBP}_{1}$ only in the $\mathrm{A}$ group $\left(\mathrm{P}<0.002\right.$ for both). $\mathrm{SBP}_{2}, \mathrm{SBP}_{3}$ and $\mathrm{ACE}$ activity showed a statistically significant increase in the $A$ vs $C(P<0.005)$, and vs AT groups $(P<0.05)$, while $\mathrm{NO}_{x}$ was significantly decreased in the $A$ and $A T$ groups vs controls $(P=0.01)$. ACE activity was strongly correlated with $\mathrm{SBP}_{3}$ in the $A$ group $(r=0.71, P=0.04)$. These findings suggest that oral supplementation of taurine may prevent the increase in $S B P$ induced by $D E C A$, an effect potentially mediated by angiotensin-converting enzyme.
\end{abstract}

Key words: Anabolic androgenic steroids; Nandrolone decanoate; Taurine; Blood pressure; Angiotensin-converting enzyme; Nitric oxide

\section{Introduction}

Anabolic androgenic steroids (AAS) are synthetic cholesterol derivatives of testosterone, designed for clinical applications in conditions such as hypogonadism, breast cancer, anemia, and various chronic catabolic states (1). Due to their anabolic effects, they are currently being used in high doses among athletes to enhance performance or in the bodybuilding community for cosmetic reasons. AAS abuse has been blamed for a large number of adverse side effects, including liver and kidney toxicity, endocrine dysfunction, psychiatric and behavioral disturbances and a variety of significant alterations on cardiovascular system with potentially severe complications (hypertension, atherosclerosis, left ventricular dysfunction, thromboembolic events, life-threatening arrhythmia, and sudden cardiac death) $(2,3)$. The continuous increase of androgen misuse and its relationship to premature death has regarded AAS abuse as a major medical and public health issue $(1,4)$. Although there is a considerable amount of evidence depicting the whole spectrum of detrimental effects of androgen abuse, data describing their association with elevated blood pressure or with the development of hypertension are still ambiguous and inconclusive $(3,4)$. Therefore, our study was designed to generate more data concerning the influence of supraphysiological administration of AAS on blood pressure in rats, and to underline possible mechanisms that may be involved. 
Taurine, often referred to as a conditionally-essential amino acid, and present in high concentrations in the brain, retina, liver, bile, myocardium, kidney, skeletal muscles and blood cells, has many beneficial effects, such as antioxidative, anti-inflammatory and anti-apoptotic activity, membrane ion exchange modulation, osmoregulation, bile acid conjugation, and neurotransmission regulation (5-7). There are multiple animal and human studies describing the protective role of taurine on the heart and vessels, suggesting that it could be useful in the prevention of cardiovascular disease (6). This sulfur-amino acid has showed several key actions on cardiovascular risk factors. It improves lipid profile, decreases plasma homocysteine, and reduces the inflammation and oxidative injuries of the intima layer of vessels, leading to a delay in the initiation and progression phases of atherosclerosis (5). Taurine also provides cardioprotection, exerts modulator effects on hemostasis, has a protective action in diabetes and its complications, and shows a beneficial influence on blood pressure $(6,8)$. Additionally, we have previously reported protective effects of taurine against the hypercoagulable state induced by suprapharmacological nandrolone decanoate (DECA) administration in rats $(9,10)$. Later, Ahmed et al. reported that taurine, by virtue of its anti-inflammatory, antioxidant and anti-apoptotic effects, prevented testicular toxicity and DNA damage induced by high doses of DECA administration in rats (11). Although taurine is also recognized for a large number of favorable actions on vasculature and it appears to be effective as a depressor agent in hypertensive situations, the involved mechanisms are not entirely clear and are still being debated $(6,12)$.

In this study, we assessed the impact of taurine administration on blood pressure, independently or in combination with high doses of DECA, and we pointed out the possible mechanisms that may underline their effects in rats. We hypothesized that this sulfur-amino acid could alleviate the blood pressure response induced by the supraphysiological DECA administration. The present experiment is the first to assess the influence of a simultaneous treatment with these two drugs on blood pressure.

\section{Material and Methods}

\section{Animals}

The study was conducted in 32 adult male Wistar rats, aged 14 weeks, with body weight $323 \pm 27 \mathrm{~g}$ (mean $\pm \mathrm{SD}$ ), housed in individual cages, floored with wood shavings, in a room with constant temperature $\left(23^{\circ} \mathrm{C}\right)$ and 12 -h lightdark cycle (lights on at 7:00 am), with access to rat chow and water ad libitum. For economic and ethical reasons, we used the minimum number of animals needed to achieve the scientific objectives. All animal procedures were carried out in accordance with the guiding principles for biomedical research involving animals, as stated by the European Communities Council Directive \#86/609/EEC and with approval of the Ethics Committee for Animal Research of the Carol Davila University of Medicine and Pharmacy (Bucharest, Romania).

\section{Chemicals}

DECA was purchased from Norma Hellas Pharmaceutical Industry (Greece, $2 \mathrm{~mL}$ Vial, $100 \mathrm{mg} / \mathrm{mL}$ ). Taurine (crystalline powder) was obtained from Merck KGaA (Germany).

\section{Experimental design}

The animals were randomly assigned to four treatment groups ( $n=8$ for each group) - androgen group (A): rats received a weekly high dose of DECA $(10 \mathrm{mg} / \mathrm{kg}$ body weight), diluted in sesame oil with benzyl alcohol $(90: 10, v / v)$ as vehicle $(15 \mathrm{mg} / \mathrm{mL})$, by intragluteal injection, for 12 weeks; taurine group $(\mathrm{T})$ : rats were supplemented with $2 \%(159.8 \mathrm{mM})$ taurine in drinking water for the same time period; animals in this group also received a weekly intramuscular injection of sesame oil with benzyl alcohol (90:10, v/v); androgen and taurine group (AT): rats treated with both DECA and taurine, in similar doses to those used in the aforementioned groups; control group (C): rats were given only weekly intramuscular vehicle injection. The DECA dose was chosen according to previous studies and was comparable to the dose that has been reported as being frequently used by abuser athletes, $600 \mathrm{mg} /$ week, or approximately $8 \mathrm{mg} \cdot \mathrm{kg}^{-1} \cdot$ week $^{-1}(13)$.

Noninvasive measurements of systolic blood pressure (SBP) were performed at the beginning of the study $\left(\mathrm{SBP}_{1}\right), 2\left(\mathrm{SBP}_{2}\right)$, and 3 months later - at the end of the study $\left(\mathrm{SBP}_{3}\right)$.

Since rat blood sampling throughout the study could interfere with blood pressure assessment and influence the results, we decided to perform the biochemical measurements only at the end of the study. The rats were euthanized by withdrawing blood from the heart, under induced and maintained anesthesia, after 12 weeks of treatment. Blood samples withdrawn by cardiac puncture were collected into heparinized plastic tubes.

\section{Noninvasive blood pressure measurement}

Systolic blood pressure was measured by tail-cuff plethysmography technique, following the BIOPAC User Guide instructions (Non-invasive Small Animal Tail Blood Pressure Systems - NIBP200A, BIOPAC Systems, Inc., USA). To condition the animal before starting the BIOPAC recordings, rats were subjected to a training session. They were placed in a suitable body restrainer several times a day, for 3 days, accompanied each time by the tail warming procedure. For accurate pressure measurement, prior to obtaining each recording, the animals were placed in the restrainer for at least 10 to $15 \mathrm{~min}$ and the tail was warmed to $32^{\circ} \mathrm{C}$. Also, a proper room temperature was maintained and a proper animal handling was carefully 
carried out. A darkened nose cone was placed over the proximal extremity of the rodent restrainer to limit the animal's view and reduce stress level. However, if the animal was under stress and moved the tail steadily, it was taken out of the restrainer and allowed to relax and become inactive before resuming the measurements and obtaining a successful SBP reading. SBP was recorded between 12:00 am and $2.30 \mathrm{pm}$. As a generally accepted method, 9 SBP measurements were performed for each rat and mean values were calculated. The lowest stable consecutive values were taken to avoid the influence of animal restlessness on SBP reading.

It would probably have been useful to assess blood pressure in physically trained rats, for an experimental model of AAS abusing athletes. However, this would have excluded other situations associated with high plasma levels of circulating androgens, such as endocrine diseases. Additionally, the model would not have reflected AAS abuse for cosmetic purposes in untrained subjects.

Plasma angiotensin-converting enzyme (ACE) activity A direct spectrophotometric assay for determining the plasma ACE activity in rat serum was used. The substrate used is a furan-acryloyl-blocked tripeptide, 2-furan-acryloylphenylalanyl-glycyl-glycine (FAPGG), which undergoes an $80 \mathrm{~nm}$ blue shift in the absorption spectrum upon hydrolysis into FA-Phe and Gly-Gly. ACE activity assays were made at $340 \mathrm{~nm}$ and $37^{\circ} \mathrm{C}$ using a measuring time of $10 \mathrm{~min}$. One unit $(U)$ of ACE activity was expressed as the amount of the enzyme that will hydrolyze $1 \mu \mathrm{mol}$ of the substrate FAPGG into FAP and glycyl-glycine in $1 \mathrm{~min}$ at $37^{\circ} \mathrm{C}(14)$. Normalized plasma ACE activity was determined at the end of the measurements, by dividing the ACE value to total plasma protein value for each animal. Total protein was assayed by Bradford method (Bio Rad Laboratories, USA). Plasma samples were diluted from an estimated initial total protein concentration of $7 \mathrm{~g} / \mathrm{dL}(70 \mathrm{mg} / \mathrm{mL})$. Absorbance was measured spectrophotometrically at $595 \mathrm{~nm}$ against a standard containing bovine serum-albumin.

\section{Plasma stable end products of NO metabolism (nitrate and nitrite)}

Nitrate and nitrite $\left(\mathrm{NO}_{\mathrm{x}}\right)$ analysis was performed spectophotometrically by an assay that combines reduction of nitrate with vanadium (III) and measurement of nitrite in a single step. Reactions were carried out at $37^{\circ} \mathrm{C}$, taking into consideration that at temperatures below $80^{\circ} \mathrm{C}$, nitrate reduction by vanadium (III) is suspended following nitrite formation. Griess reagents [N-1-naphthylethylenediamine dihydrochloride (NEDD) and sulphanilamide (SULF)] were used as trapping agents for simultaneous detection of nitrate and nitrite. A nitrate standard solution $(50 \mu \mathrm{L}$ ) was serially diluted (from 100 to $5 \mu \mathrm{M}$ ) in duplicate in a 96-well flat-bottomed, polystyrene microtiter plate (Corning, USA). After loading the plate with samples $(50 \mu \mathrm{L})$, addition of a saturated solution of $\mathrm{VCl}_{3}(40 \mathrm{mg}$ in
$5 \mathrm{~mL} 1 \mathrm{M} \mathrm{HCl})$ to each well $(50 \mu \mathrm{L})$ was immediately followed by addition of SULF $2 \%$ in $1 \mathrm{M} \mathrm{HCl}(25 \mu \mathrm{L})$ and $0.1 \%$ NEDD in $\mathrm{dH}_{2} \mathrm{O}(25 \mu \mathrm{L})$. The samples were incubated for $40 \mathrm{~min}$ at $37^{\circ} \mathrm{C}$, and nitrate content was measured spectrophotometrically at $540 \mathrm{~nm}$. The $\mathrm{VCl}_{3}$ solution and the Griess reagents were freshly prepared immediately prior to application to the plate. Sample blank values were obtained by substituting diluting medium for Griess reagent. Nitrite was similarly measured, except that samples and nitrite standards were only exposed to Griess reagents (15).

\section{Statistical analysis}

The normal probability distribution was tested using the Kolmogorov-Smirnov test. Continuous data are reported as means $\pm S D$. One-way ANOVA followed by Tukey's post hoc analysis was performed for comparisons between groups regarding biochemical parameters and body weight. General linear model (repeated measures) was used to compare the intergroup and intragroup variations of blood pressure, followed by Bonferroni's post hoc test. The relationship between different parameters was assessed by Pearson's method. SPSS (Statistical Package for Social Sciences, Inc., USA) Windows 20.0 software was used for statistical analysis. A two-sided $\mathrm{P}<0.05$ was considered to be statistically significant.

\section{Results}

Body weight was similar in the four groups of rats at the beginning of the experiment $(P=0.55)$. At the end of the study the increase in body weight was smaller in the A, T and AT groups (Table 1), although the differences were not significant from the controls $(P=0.06)$.

\section{Effects of DECA and taurine on blood pressure}

During the experiment, systolic blood pressure significantly changed only in the A group (intragroup variation). In this group, $\mathrm{SBP}_{2}$ and $\mathrm{SBP}_{3}$ significantly increased compared to $\mathrm{SBP}_{1}\left(\mathrm{SBP}_{2}\right.$ vs $\mathrm{SBP}_{1}, \mathrm{P}=0.002$, SBP mean difference $=5.9 \mathrm{mmHg}, 95 \%$ confidence interval $(\mathrm{Cl})=$ 1.95-9.84 and $\mathrm{SBP}_{3}$ vs $\mathrm{SBP}_{1}, \mathrm{P}<0.001$, SBP mean difference=7.52 $\mathrm{mmHg} ; 95 \% \mathrm{Cl}=3.48-11.56$ ) (Figure 1).

A significant difference was found in SBP among the 4 groups of rats (intergroup variation) at 2 and 3 months after the beginning of the study $(P<0.001)$. Post hoc analysis showed that SBP significantly increased in the A vs $\mathrm{C}$ group, at both $2(\mathrm{P}=0.001$, SBP mean difference $=$ $12.6 \mathrm{mmHg} ; 95 \% \mathrm{Cl}=3.99-21.21)$ and 3 months $(\mathrm{P}<0.001$, SBP mean difference $=16.07 \mathrm{mmHg} ; 95 \% \mathrm{Cl}=8.75-23.39$ ) . A significant difference was also found in the A vs AT group at both $2(\mathrm{P}=0.007$, SBP mean difference $=10.95 \mathrm{mmHg}$; $95 \% \mathrm{Cl}=2.34-19.56)$ and 3 months $(\mathrm{P}<0.001$, SBP mean difference $=12.27 \mathrm{mmHg} ; 95 \% \mathrm{Cl}=4.95-19.59)$. No significant differences were found in SBP between the Tand $\mathrm{C}$ 
Table 1. Values of systolic blood pressure, biochemical parameters and body weight in rats treated with nandrolone decanoate $(A)$, taurine $(T)$ and their combination $(A T)$, or with vehicle $(C)$.

\begin{tabular}{lccccc}
\hline Variable & C group & A group & T group & AT group & $P$ \\
\hline $\mathrm{SBP}_{1}(\mathrm{mmHg})$ & $113.8 \pm 6.8$ & $116.8 \pm 7.2$ & $114.1 \pm 5.8$ & $114.8 \pm 6.1$ & $\mathrm{~ns}$ \\
$\mathrm{SBP}_{2}(\mathrm{mmHg})$ & $111.1 \pm 6.9$ & $134.5 \pm 10.3$ & $116.2 \pm 5.8$ & $121.3 \pm 12.9$ & $<0.001$ \\
$\mathrm{SBP}_{3}(\mathrm{mmHg})$ & $115.2 \pm 5.4$ & $136.8 \pm 10.5$ & $111.5 \pm 8.2$ & $123.3 \pm 10.5$ & $<0.001$ \\
$\mathrm{ACE}_{\text {activity }}(\mathrm{U} / \mathrm{g})$ & $7.7 \pm 1.5$ & $11.3 \pm 2.1$ & $7.3 \pm 2.0$ & $8.6 \pm 2.0$ & 0.001 \\
$\mathrm{NO}_{\mathbf{x}}(\mu \mathrm{mol} / \mathrm{L})$ & $322.1 \pm 70.3$ & $233.2 \pm 47.6$ & $292.6 \pm 43.9$ & $235.4 \pm 51.6$ & 0.005 \\
$\mathrm{BW}_{\mathrm{i}}(\mathrm{g})$ & $332.5 \pm 33.2$ & $321.2 \pm 30.9$ & $313.7 \pm 15.9$ & $323.7 \pm 19.9$ & $\mathrm{~ns}$ \\
$\mathrm{BW}_{\mathrm{f}}(\mathrm{g})$ & $395 \pm 28.2$ & $382.5 \pm 36.5$ & $392.5 \pm 11.6$ & $362.5 \pm 16.6$ & $\mathrm{~ns}$ \\
\hline
\end{tabular}

Data are reported as means $\pm \mathrm{SD}\left(\mathrm{n}=8\right.$ /group). $\mathrm{SBP}_{1}, \mathrm{SBP}_{2}, \mathrm{SBP}_{3}$ : systolic blood pressure at the beginning of the study, at 2, and 3 months (at the end of the study), respectively; $A C E_{\text {activity: normalized plasma angiotensin }}$ converting enzyme activity; $\mathrm{NO}_{\mathrm{x}}$ : plasma stable end products of $\mathrm{NO}$ metabolism - nitrate and nitrite; $\mathrm{BW}_{\mathrm{i}}$ : initial body weight; $\mathrm{BW}_{\mathrm{f}}$ : final body weight. ANOVA was used for analyses of $\mathrm{ACE}_{\text {activity, }} \mathrm{NO}_{\mathrm{x}}$ and $\mathrm{BW}$; General Linear Model (repeated measures) for SBP; ns: not significant.

groups, or between the AT and $\mathrm{C}$ groups, at 2 or 3 months $(P>0.93$; Figure 1).

\section{Effects of DECA and taurine on plasma ACE activity}

A significant difference was found concerning ACE activity $(P=0.001$, Table 1$)$. Post hoc analysis revealed a statistically significant increase in the $A$ vs $C$ group $(P=0.004)$ and in the $A$ vs AT group $(P=0.04)$, while between the AT and $C$ groups, or $C$ and $T$ groups there were no significant differences (Figure 2). In the A group $A C E$ activity was strongly correlated with $\mathrm{SBP}_{3}(r=0.71$, $\mathrm{P}=0.04$ ), but not with $\mathrm{SBP}_{2}$. ACE activity also tended to be related to $\mathrm{SBP}_{3}$ in the $\mathrm{AT}$ group, but without reaching statistical significance $(r=0.63, P=0.08)$.

\section{Effects of DECA and taurine on plasma stable end products of NO metabolism (nitrate and nitrite)}

Mean values for plasma levels of NO stable metabolic end products - nitrate and nitrite - are reported in Table 1.
There was a significant difference concerning $\mathrm{NO}_{\mathrm{x}}$ $(\mathrm{P}=0.005)$. Post hoc analysis of $\mathrm{NO}_{x}$ showed a significant decrease in the A and AT groups vs the $C$ group $(P=0.01)$, while no significant differences were registered between the A and AT groups, or between the $\mathrm{C}$ and $\mathrm{T}$ groups (Figure 3). There were no significant correlations between $\mathrm{NO}_{x}$ and $\mathrm{SBP}_{2}$ or $\mathrm{SBP}_{3}$ in any group.

\section{Discussion}

The present study brings complementary data regarding the action of DECA and taurine on blood pressure, a field of interest still dominated by controversial data. It also shows the cumulative effect of the two drugs on blood pressure for the first time. We found that chronic, suprapharmacological DECA administration induced a pressor response in rats, which was prevented by concomitant taurine administration. DECA also increased ACE activity and decreased $\mathrm{NO}_{x}$ levels, suggesting that it may partially contribute to blood

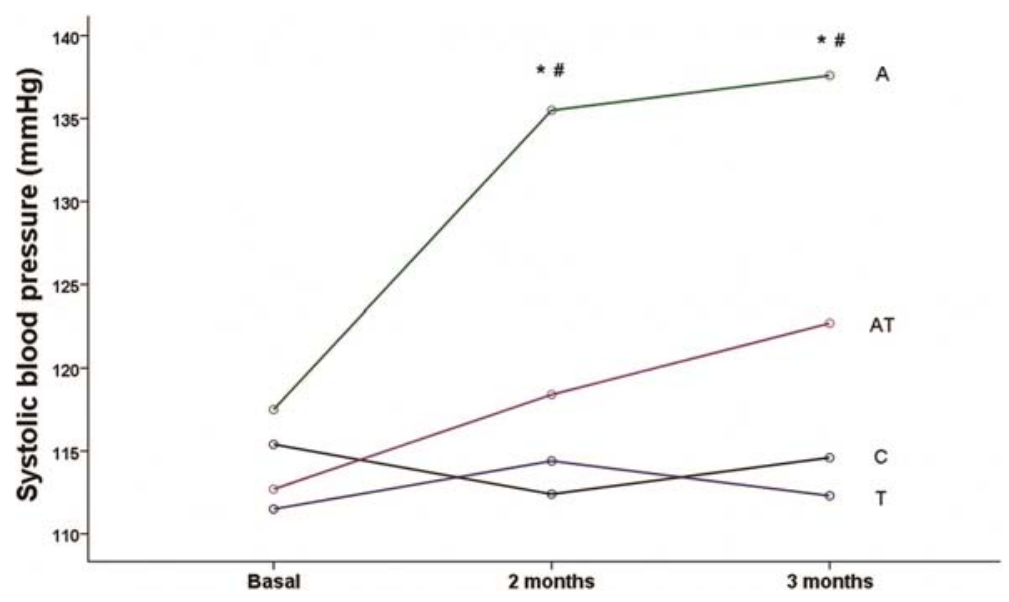

Braz J Med Biol Res | doi: 10.1590/1414-431X20165116
Figure 1. Effect of nandrolone decanoate (A), taurine $(\mathrm{T})$ and their combination (AT) on systolic blood pressure (SBP) of rats. There was a significant increase of SBP at 2 and 3 months vs basal SBP only in the A group. SBP was significantly higher in the $A$ vs control $(C)$ group and also in the A vs AT group ( $n=8$ for each group), at 2 and 3 months (General Linear Model; repeated measures; and Bonferroni's post hoc test). ${ }^{*} \mathrm{P} \leqslant 0.002$ vs basal blood pressure; ${ }^{\#} \mathrm{P} \leqslant 0.007$ vs the $\mathrm{C}$ and $\mathrm{AT}$ groups. 
pressure elevation in the androgen treated group. Chronic taurine supplementation in drinking water had no effect in intact rats, but significantly decreased ACE activity in the mixed treated group, thus offering a potential explanation for the blood pressure change in this group of rats.

\section{Effects of DECA on blood pressure}

Our findings related to the influence of supraphysiological AAS administration on noninvasively measured systolic blood pressure support those of several other animal studies $(16,17)$. In humans, increased blood pressure, or even hypertension has been linked to high levels of circulating androgens, induced either by various endocrinological diseases [polycystic ovary syndrome (PCOS), adrenogenital syndrome, virilizing gonadal tumors, enzyme defects, medication induced hyperandrogenism] $(18,19)$, or by AAS abuse in athletes or amateur bodybuilders $(1,2)$. However, there is still a controversy regarding the pressor effects of AAS misuse in the sporting environment $(3,4)$. The currently available data, coming mostly from observational studies, indicate either an elevation of blood pressure (which can persist for up to 1 year after drug intake cessation) $(2,20,21)$ or no significant blood pressure changes in AAS users $(3,22,23)$. The variety of outcomes may result from the lack of homogeneity in type, dosage, and timing of AAS administration, exercise regimen and from the difficulty in controlling drug abuse in both the AAS and control groups (1).

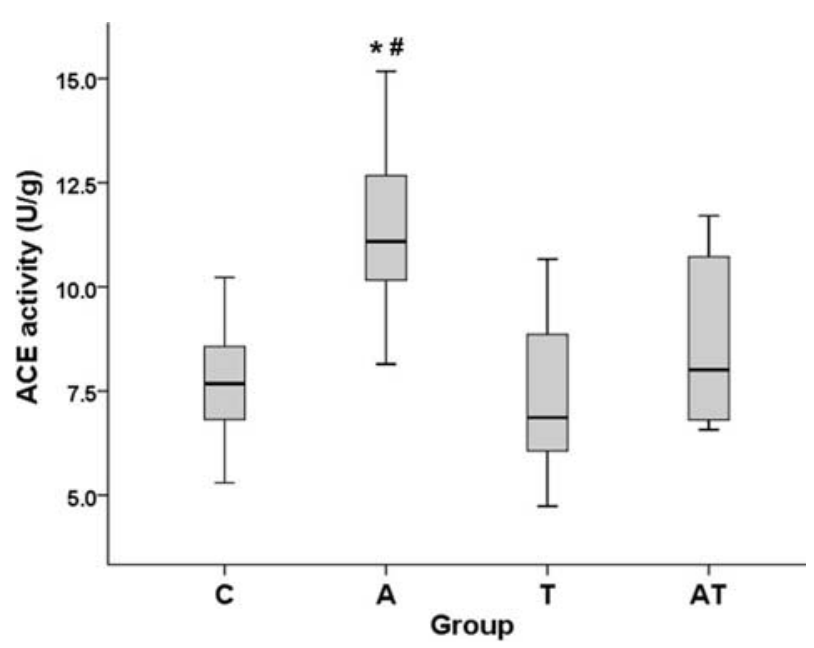

Figure 2. Effect of nandrolone decanoate $(A)$, taurine $(T)$ and their combination (AT) on plasma angiotensin converting enzyme (ACE) activity in rats. There was a significant increase of ACE activity in the A vs control (C) group and also $v s$ the AT group $(n=8$ for each group). The box-and-whisker plots report the minimum, $25 \%$ percentile, median, $75 \%$ percentile, and maximum values. ${ }^{*} \mathrm{P}=0.004$ vs $\mathrm{C}$ group; ${ }^{\#} \mathrm{P}=0.04$ vs AT group. (one-way ANOVA and Tukey's post hoc analysis).
The increase of plasma ACE activity in the DECA treated group is also consistent with published data. It has been previously proven that androgens have a stimulatory action on renin-angiotensin system (RAS), upregulating the angiotensinogen and renin mRNA expression, increasing angiotensin II and plasma renin activity, and enhancing the angiotensin type 1 receptor pathways (24-26). Moreover, high levels of plasma androgens increased the renal cortical expression of $A C E$ in a rat model of PCOS (26). DECA, at doses twice the one we used, $20 \mathrm{mg} \cdot \mathrm{kg}^{-1} \cdot$ week $^{-1}$, was shown to induce an elevation of cardiac tissue ACE activity in untrained rats (16). The close correlation between ACE activity and $\mathrm{SBP}_{3}$ identified in our study allowed us to consider the ACE pathway as one of the possible mechanisms underlying SBP variation in the DECA group.

Regarding the plasma stable end products of $\mathrm{NO}$ metabolism, nitrate and nitrite, the decrease of $\mathrm{NO}_{\mathrm{x}}$ level in the DECA group could also explain the increase of the SBP in this group vs controls. These findings are consistent with previous data. It was proven that in humans supraphysiological doses of androgens could decrease the endothelial NO synthase (eNOS) expression (27). Also, DECA administered in a dose commonly used by heavy AAS abusers was shown to decrease the level of aortic eNOS in rats (28). On the other hand, there are data supporting the view that androgens may have a stimulatory effect on NO production, as was noted in experiments performed on the rat Leydig cell

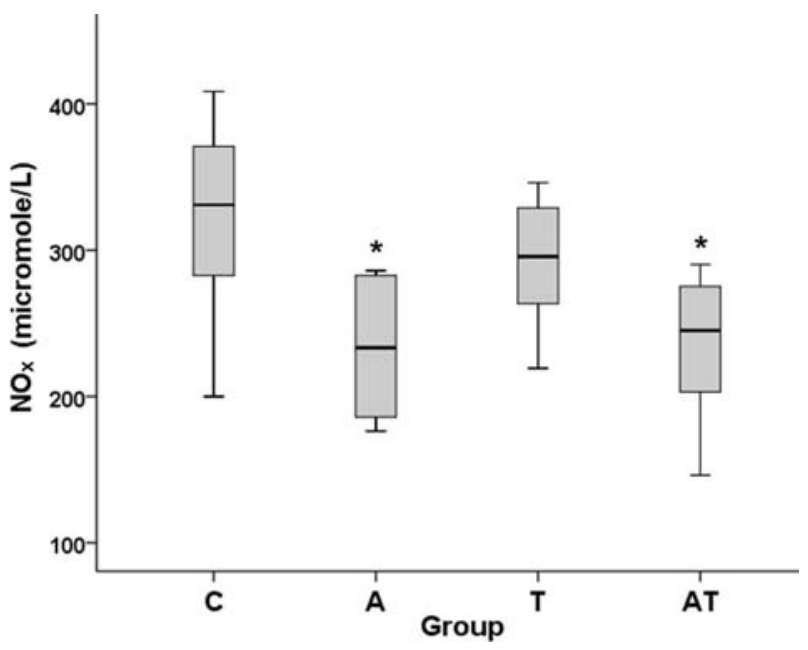

Figure 3. Effect of nandrolone decanoate $(A)$, taurine $(T)$ and their combination (AT) on plasma levels of stable end products of NO metabolism - nitrate and nitrite $\left(\mathrm{NO}_{\mathrm{x}}\right)$ - in rats. There was a significant decrease of $\mathrm{NO}_{x}$ in the A and AT groups vs control (C) group ( $n=8$ for each group). The box-and-whisker plots report the minimum, $25 \%$ percentile, median, $75 \%$ percentile, and maximum values. ${ }^{*} \mathrm{P}=0.01$ vs the $\mathrm{C}$ group (one-way ANOVA and Tukey's post hoc analysis). 
culture or testicular tissue sample $(11,29)$. A possible explanation for plasma NO decrease exerted by androgens, as obtained in the present study, has been given by Reckelhoff et al. (24) and afterwards by Xue et al. (25), who promoted the opinion that androgens could induce blood pressure elevation by superoxide concentration increase. The cause of this increase is the enhancement of the oxidative stress generated by androgens, either directly or indirectly, via RAS stimulation and angiotensin II release. Increased superoxide anion can subsequently interact with NO, leading to peroxynitrite formation, one of the most potent oxidative compounds and a substance with a powerful vasoconstrictor action induced through multiple mechanisms $(24,25)$. Considering all the existent controversial data and hypothesis, our study brings an additional argument to the detrimental action of AAS on NO production when administered in supraphysiological doses. This could reflect the presence of endothelial dysfunction, one of the cardiovascular side-effects often reported following AAS misuse.

\section{Effects of taurine on blood pressure}

As it is revealed by the noninvasive pulse plethysmographic assessment, taurine exerted no influence on systolic blood pressure in intact rats, but significantly decreased its level at 2 and 3 months after administration in the mixed treated animals. This effect is consistent with the antihypertensive action of sulfur-amino acid, evidenced in animal studies using hypertensive models, and suggested by several clinical and epidemiological studies $(5,6,8)$. On the other hand, accumulating evidence from laboratory experiments showed that oral taurine supplementation does not seem to affect blood pressure in healthy animals (6). The described inhibitory effect of the sulfur-amino acid in the hypertension setting and the neutral effect in intact animals may be of worth, as taurine was able to stabilize the disorder and further preserve the homeostasis. In fact, based on its protective role on the cardiovascular system, taurine has been approved in Japan as a therapeutic agent for heart failure treatment (30).

Taurine was effective in lowering the increased level of ACE activity induced by nandrolone, while exerting no action in intact rats. A tendency for correlation has been found between ACE activity and $\mathrm{SBP}_{3}$ in the mixed treated group. Our findings didn't establish a causative mechanism by which taurine exerts its benefits, but one possible explanation for the blood pressure reduction in the mixed treated group of rats might be its action on ACE. Several lines of evidence support our results related to taurine influence on ACE activity. It has been previously proven that taurine attenuates renin-angiotensin system overactivity, preventing blood pressure elevation induced by renin, potentiating the effects of ACE inhibitors, modulating the expression of $\mathrm{AT}_{2}$ receptors, and antagonizing the harmful effects of angiotensin II on the heart, blood vessels and kidneys $(8,30,31)$. However, scarce data point out the influence of the sulfur-amino acid on altered ACE activity caused by several pathological conditions $(32,33)$. In these studies taurine exerted no influence on ACE activity in intact rats, as we also found. This can be assigned to the generally observed behavior of the sulfur-amino acid, to act only on drug-induced organ or systemic pathophysiology (34).

Taurine presented a slight propensity to decrease plasma NO stable end products in intact rats, without reaching statistical significance. The neutral influence of taurine on NO metabolism in healthy animals has been cited in the literature $(11,32)$, despite the fact that a vasodilator effect of the sulfur-amino acid has been systematically proven (6). The also neutral effect on plasma $\mathrm{NO}_{x}$ levels obtained in the mixed treated group is quite surprising, considering the reduction of blood pressure in this group. This is even more striking when taking into account the considerable amount of available data concerning the stimulatory action of taurine on $\mathrm{NO}$ production $(5,6)$. On the other hand, data from experiments conducted on animal models or cell cultures also reveal an inhibiting action of sulfur-amino acid on NO production (35-37). It has been suggested that this influence may be linked to the taurine's ability to quench $\mathrm{NO}$, which along the proved inhibitory effect on superoxide generation, can prevent reactive nitrogen species formation - i.e., peroxynitrite $(11,37)$. The antioxidant activity of the sulfur-amino acid is actually quite well stated $(5,31)$. Recently, taurine has been shown to exhibit protective effects against heavy exercise-induced nitrosative inflammation and stress, preventing inducible nitric oxide synthase (iNOS) expression in rats skeletal muscle (38), which may be of interest considering the stimulatory action of supraphysiological doses of AAS on oxidative stress. Overall, the influence of taurine on the process of NO synthesis and release is still controversial. The current contradictory data are probably dependent on the large variety of study designs. In this context, complementary data regarding this field of investigation could be valuable.

The present findings led us to the conclusion that taurine may have the ability to protect against a panel of insults generated by AAS administration, but it fails to exhibit any influence on healthy animals. Oral supplementation of the sulfur-amino acid restored the nandrolone-induced increased values of SBP. This effect may be explained by the detrimental influence of DECA on ACE and $\mathrm{NO}_{\mathrm{x}}$, and by the beneficial influence of taurine on ACE. Our study brings complementary data into a very exciting area of interest, which still has a knowledge gap and controversy over the possible mechanisms through which the two drugs may influence blood pressure. Extrapolating to humans, this study challenges the assumption that taurine, a substance safely used for 
more than two decades, may be useful in several circumstances associated with high levels of circulating androgens. It remains to be established if this sulfuramino acid may have an effective role in individuals abusing of AAS, with poor compliance to standard antihypertensive therapies and to the physician's AAS withdrawal recommendation. This could be of interest especially because taurine is often used to enrich beverages with the purpose of boosting athletic performance. Certainly, prospective studies with long-term taurine use are needed in order to verify these hypotheses and to clarify the exact mechanisms that may underline these effects.

\section{References}

1. Achar S, Rostamian A, Narayan SM. Cardiac and metabolic effects of anabolic-androgenic steroid abuse on lipids, blood pressure, left ventricular dimensions, and rhythm. Am J Cardiol 2010; 106: 893-901, doi: 10.1016/j.amjcard. 2010.05.013

2. Santos MA, Oliveira CV, Silva AS. Adverse cardiovascular effects from the use of anabolic-androgenic steroids as ergogenic resources. Subst Use Misuse 2014; 49: 1132-1137, doi: 10.3109/10826084.2014.903751

3. Vanberg P, Atar D. Androgenic anabolic steroid abuse and the cardiovascular system. Handb Exp Pharmacol 2010; 411-457, doi: 10.1007/978-3-540-79088-4_18

4. Nieschlag E, Vorona E. Doping with anabolic androgenic steroids (AAS): Adverse effects on non-reproductive organs and functions. Rev Endocr Metab Disord 2015; 16: 199-211, doi: 10.1007/s11154-015-9320-5

5. Murakami S. Taurine and atherosclerosis. Amino Acids 2014; 46: 73-80, doi: 10.1007/s00726-012-1432-6

6. Abebe W, Mozaffari MS. Role of taurine in the vasculature: an overview of experimental and human studies. Am $J$ Cardiovasc Dis 2011; 1: 293-311.

7. Zhang LY, Zhou YY, Chen F, Wang B, Li J, Deng YW, et al. Taurine inhibits serum deprivation-induced osteoblast apoptosis via the taurine transporter/ERK signaling pathway. Braz J Med Biol Res 2011; 44: 618-623, doi: 10.1590/ S0100-879X2011007500078

8. Xu YJ, Arneja AS, Tappia PS, Dhalla NS. The potential health benefits of taurine in cardiovascular disease. Exp Clin Cardiol 2008; 13: 57-65.

9. Rosca AE, Badiu C, Uscãtescu V, Stoian I, Miricã R, Braga $\mathrm{RI}$, et al. Influence of chronic administration of anabolic androgenic steroids and taurine on haemostasis profile in rats: a thrombelastographic study. Blood Coagul Fibrinolysis, 2013; 24: 256-260, doi: 10.1097/MBC.0b013e32835 b7611

10. Rosca A, Badiu C, Uscatescu V, Mirica R, Bragam R, Pavel $B$, et al. Effect of chronic administration of anabolic androgenic steroids and taurine on platelet aggregation in rats. Acta Endocrinologica 2013; 9: 33-38, doi: 10.4183/ aeb.2013.33

11. Ahmed MA. Amelioration of nandrolone decanoate-induced testicular and sperm toxicity in rats by taurine: effects on steroidogenesis, redox and inflammatory cascades, and

\section{Acknowledgments}

The authors wish to thank Dr. Coman Cristin, Dr. Marinescu Bogdan, and Mr. Pinzari Gheorghe for laboratory assistance in animal care and experiments. This paper was partly supported by the Sectorial Operational Program for Human Resources Development (SOPHRD), and financed by the European Social Fund and the Romanian Government under the contract No. POSDRU 141531/2014. Paper publication is supported by the research grant No. PN-II-PT-PCCA-2013-41851, contract $192 / 2014$, financed by the Romanian Ministry of National Education and Scientific Research.

intrinsic apoptotic pathway. Toxicol Appl Pharmacol 2015; 282: 285-296, doi: 10.1016/j.taap.2014.12.007

12. Suwanich A, Wyss JM, Roysommuti S. Taurine supplementation in spontaneously hypertensive rats: Advantages and limitations for human applications. World J Cardiol 2013; 5: 404-409, doi: 10.4330/wjc.v5.i11.404

13. Norton GR, Trifunovic B, Woodiwiss AJ. Attenuated beta-adrenoceptor-mediated cardiac contractile responses following androgenic steroid administration to sedentary rats. Eur J Appl Physiol 2000; 81: 310-316, doi: 10.1007/ s004210050048

14. Harjanne A. Automated kinetic determination of angiotensinconverting enzyme in serum. Clin Chem 1984; 30: 901-902.

15. Miranda KM, Espey MG, Wink DA. A rapid, simple spectrophotometric method for simultaneous detection of nitrate and nitrite. Nitric Oxide 2001; 5: 62-71, doi: 10.1006/ niox.2000.0319

16. Franquni JV, do Nascimento AM, de Lima EM, Brasil GA, Heringer OA, Cassaro KO, et al. Nandrolone decanoate determines cardiac remodelling and injury by an imbalance in cardiac inflammatory cytokines and ACE activity, blunting of the Bezold-Jarisch reflex, resulting in the development of hypertension. Steroids 2013; 78: 379-385, doi: 10.1016/ j.steroids.2012.12.009

17. das Neves V, Tanno AP, Cunha TS, Fernandes T, Guzzoni $V$, da Silva CA, et al. Effects of nandrolone and resistance training on the blood pressure, cardiac electrophysiology, and expression of atrial beta-adrenergic receptors. Life Sci 2013; 92: 1029-1035, doi: 10.1016/j.lfs.2013.04.002

18. Bjorntorp P. The android woman - a risky condition. $J$ Intern Med 1996; 239: 105-110, doi: 10.1046/j.1365-2796.1996. 364690000.x

19. Azziz R, Nestler JE, Dewailly D. Androgen excess disorders in women. 2nd edn. New Jersey: Humana Press Inc.; 2007, doi: 10.1007/978-1-59745-179-6

20. Riebe D, Fernhall B, Thompson PD. The blood pressure response to exercise in anabolic steroid users. Med Sci Sports Exerc 1992; 24: 633-637, doi: 10.1249/00005768199206000-00004

21. Urhausen A, Albers T, Kindermann W. Are the cardiac effects of anabolic steroid abuse in strength athletes reversible? Heart 2004; 90: 496-501, doi: 10.1136/ hrt.2003.015719 
22. Kuipers H, Wijnen JA, Hartgens F, Willems SM. Influence of anabolic steroids on body composition, blood pressure, lipid profile and liver functions in body builders. Int $J$ Sports Med 1991; 12: 413-418, doi: 10.1055/s-2007-1024704

23. D'Andrea A, Caso P, Salerno G, Scarafile R, De Corato G, Mita $C$, et al. Left ventricular early myocardial dysfunction after chronic misuse of anabolic androgenic steroids: a Doppler myocardial and strain imaging analysis. Br J Sports Med 2007; 41: 149-155, doi: 10.1136/bjsm.2006.030171

24. Reckelhoff JF. Gender differences in the regulation of blood pressure. Hypertension 2001; 37: 1199-1208, doi: 10.1161/ 01.HYP.37.5.1199

25. Xue B, Johnson AK, Hay M. Sex differences in angiotensin II-induced hypertension. Braz J Med Biol Res 2007; 40: 727-734, doi: 10.1590/S0100-879X2007000500018

26. Yanes LL, Romero DG, Moulana M, Lima R, Davis DD, Zhang $\mathrm{H}$, et al. Cardiovascular-renal and metabolic characterization of a rat model of polycystic ovary syndrome. Gend Med 2011; 8: 103-115, doi: 10.1016/j.genm.2010.11.013

27. Skogastierna C, Hotzen M, Rane A, Ekstrom L. A supraphysiological dose of testosterone induces nitric oxide production and oxidative stress. Eur J Prev Cardiol 2014; 21: 1049-1054, doi: $10.1177 / 2047487313481755$

28. Sun M, Shen W, Zhong M, Wu P, Chen H, Lu A. Nandrolone attenuates aortic adaptation to exercise in rats. Cardiovasc Res 2013; 97: 686-695, doi: 10.1093/cvr/cvs423

29. Janjic MM, Stojkov NJ, Andric SA, Kostic TS. Anabolicandrogenic steroids induce apoptosis and NOS2 (nitricoxide synthase 2 ) in adult rat Leydig cells following in vivo exposure. Reprod Toxicol 2012; 34: 686-693, doi: 10.1016/ j.reprotox.2012.10.003

30. Schaffer SW, Ito T, Azuma J. Clinical significance of taurine. Amino Acids 2014; 46: 1-5, doi: 10.1007/s00726-013-1632-8
31. Ito T, Schaffer S, Azuma J. The effect of taurine on chronic heart failure: actions of taurine against catecholamine and angiotensin II. Amino Acids 2014; 46: 111-119, doi: 10.1007/ s00726-013-1507-z

32. Hu J, Xu X, Yang J, Wu G, Sun C, Lv Q. Antihypertensive effect of taurine in rat. Adv Exp Med Biol 2009; 643: 75-84, doi: 10.1007/978-0-387-75681-3

33. Nandhini AT, Thirunavukkarasu V, Anuradha CV. Potential role of kinins in the effects of taurine in high-fructose-fed rats. Can J Physiol Pharmacol 2004; 82: 1-8, doi: 10.1139/y03-118

34. Das J, Roy A, Sil PC. Mechanism of the protective action of taurine in toxin and drug induced organ pathophysiology and diabetic complications: a review. Food Funct 2012; 3: 1251-1264, doi: 10.1039/c2fo30117b

35. Redmond HP, Wang JH, Bouchier-Hayes D. Taurine attenuates nitric oxide- and reactive oxygen intermediatedependent hepatocyte injury. Arch Surg 1996; 131: 12801287, doi: 10.1001/archsurg.1996.01430240034004

36. Sun M, Zhao Y, Gu Y, Xu C. Anti-inflammatory mechanism of taurine against ischemic stroke is related to downregulation of PARP and NF-kappaB. Amino Acids 2012; 42: 1735-1747, doi: 10.1007/s00726-011-0885-3

37. Oliveira MW, Minotto JB, de Oliveira MR, Zanotto-Filho A, Behr GA, Rocha RF, et al. Scavenging and antioxidant potential of physiological taurine concentrations against different reactive oxygen/nitrogen species. Pharmacol Rep 2010; 62: 185-193, doi: 10.1016/S1734-1140(10) 70256-5

38. Sugiura H, Okita S, Kato T, Naka T, Kawanishi S, Ohnishi S, et al. Protection by taurine against INOS-dependent DNA damage in heavily exercised skeletal muscle by inhibition of the NF-kappaB signaling pathway. Adv Exp Med Biol 2013; 775: 237-246, doi: 10.1007/978-1-4614-6130-2 